\title{
PD-L1/PD-L2 Peptide-Montanide Vaccine
}

National Cancer Institute

\section{Source}

National Cancer Institute. PD-L1/PD-L2 Peptide-Montanide Vaccine. NCI Thesaurus. Code C154564.

A vaccine composed of peptides derived from the immune checkpoint molecules, programmed death lig and 1 (PD-L1) and programmed death lig and 2 (PD-L2), combined with the immunoadjuvant montanide ISA-51, with potential immunomodulating and antineoplastic activities. Vaccination with PD-L1/PD-L2 peptide-montanide vaccine may stimulate an immune response against PD-L1 and PD-L2 expressing cells. This may enhance T-cell proliferation, cytokine production, and T-cell mediated cytolysis. Binding of programmed cell death protein 1 (PD-1; PDCD1; CD279) by its ligands, PD-L1 and PD$L 2$, results in downregulation of $\mathrm{T}$-cell responses and enhanced immune evasion. Montanide ISA-51, also known as incomplete Freund's adjuvant or IFA, is a stabilized water-in-oil emulsion adjuvant containing mineral oil with mannide oleate added as a surfactant that non-specifically stimulates cell-mediated immune responses to antigens. 